\title{
34 \\ Patients as regulatory actors in their own health care
}

\author{
Judith Healy
}

\section{Introduction}

This chapter discusses the ways in which an individual patient can be a regulatory actor in ensuring that the treatment he/she receives is safe and of good quality. While health policies now promote patients as regulatory actors, this is not an easy role for patients to undertake. This is so for several reasons, including the weight of knowledge and power inherent in health professionals (particularly doctors) compared with patients. Building on my earlier work (Healy 2011a; Healy 2011b: Chapter 9; Healy and Dugdale 2009), this chapter addresses the following regulatory concepts, dilemmas and questions:

- Why regulate: What is the rationale for patients to act as regulators?

- Information asymmetry: Do patients have sufficient information to regulate?

- Power asymmetry: What powers can patients exert through pyramids of supports and sanctions?

- Networked regulation: Who can patients enlist to strengthen their influence?

- Transparency: Are patients told when things go wrong?

- Trust: Does information erode therapeutic confidence in one's doctor? 
To begin with the first question, why should policymakers enlist patients as regulators and what is the justification for stronger regulation in health care? Virtually everyone during their lifetime consults health professionals, takes medication and undergoes procedures. The main reason for engaging people in regulating their own health care, therefore, is the pragmatic one that we are the ones who suffer most keenly the consequences of unsafe or poor-quality health care. From a patient's point of view (and we are all patients), we should have a say; we are too trusting in leaving it all to the doctors.

The complacent assumption about the safety and quality of modern health care has been shaken over the past decade. Health care can be a risky business. Studies from several high-income countries, including Australia (Runciman et al. 2000), report that between 4 and 12 per cent of patients experience an adverse event in hospital—in other words, you, as a patient, have about a one in 10 chance of something going wrong. Even in the best of hospitals, therefore, some form of unintended error can occur in a person's care. Further, substandard health services are not uncommon. For example, a population-level survey of adult patients in the United States found that only about half had received the treatment recommended in clinical protocols (McGlynn et al. 2003).

The concept of a patient as a regulatory actor invokes the principle of personal responsibility. But is it fair to ask people to take more responsibility for the quality and safety of their own health care? Patients are only one type of regulatory actor in a complex healthcare system, and health authorities are in the process of strengthening governance by a range of actors. Clearly, there are limitations to expecting sick people to engage as regulatory actors given lessened capacity during illness. There are also other barriers to participation including a class barrier of fewer resources, both educational and financial, a cultural barrier of different expectations and language, a knowledge barrier given the esoteric nature of medical expertise and a power barrier given the unequal relationship between doctors and patients. Nevertheless, some of these barriers can be surmounted, and this chapter goes on to discuss such strategies. 


\section{Concepts of patients}

The term 'patient' is used in this chapter mainly because it is difficult to find another appropriate word. While 'patient' implies passivity (contrary to the argument here), alternative words also carry dubious connotations, such as 'consumer' and 'customer'. The last two terms imply that health care is entirely a commodity rather than a public good-a value position that is not endorsed in this chapter.

The metaphors of 'exit' and 'voice' suggest how individuals can influence service providers (Hirschman 1970). 'Voice' is a political concept that fits a citizen participation paradigm, referring to the ability of a person to influence a service provider while continuing to use the service. This assumes an active not a passive person who can ask for information, negotiate options, fill out surveys, use complaint procedures and join an interest group. 'Exit' is an economic concept that fits a market model paradigm and refers to a person's ability to leave a service and go elsewhere, which assumes that a person has the capacity to leave a service, that other options are available and that the person is able to obtain or pay for another service and can make an informed choice-all of which are somewhat problematic assumptions in health care.

This chapter draws on the model of responsive regulation developed by John Braithwaite and colleagues, and the regulatory pyramid in particular, since this offers a framework for considering actors and strategies (see John Braithwaite, Chapter 7, this volume). The users of health services can be conceptualised as regulatory actors in terms of six roles (and accompanying strategies): informed patients, selective consumers, vocal complainants, entitled citizens, active partners and aggrieved litigants. The ways in which a patient can exert influence draw on mechanisms that range upwards in a regulatory pyramid, from voluntary strategies at the base (asking for information, giving consent), to market strategies (responding to a survey, making a complaint), to enforcement strategies (asserting one's rights, suing for malpractice). These concepts are depicted in Figure 34.1, with informed patients at the wide base of the pyramid (since this strategy is most commonly used) rising to active partners and aggrieved litigants towards the apex. 
This pyramid is a hybrid form of two pyramids: a sanctions-based and a strengths-based pyramid (Braithwaite et al. 2007: 319; Braithwaite 2009: 28). 'Informed patients' and 'active partners', for example, draw on the strengths of patients themselves in supporting a good standard of health care by being better informed and by participating in decisions. 'Vocal complainants' and 'aggrieved litigants', in contrast, are examples of patients who invoke sanctions against poor services and professional malpractice. Regulators could devise two pyramids-supports and sanctions - and offer a range of mechanisms that patients might use from base to apex. Further, the pyramid can be embellished with the addition of network partners at different levels (Braithwaite 2009: 30), whereby patients seek to enlist others in their efforts to obtain goodquality health care.

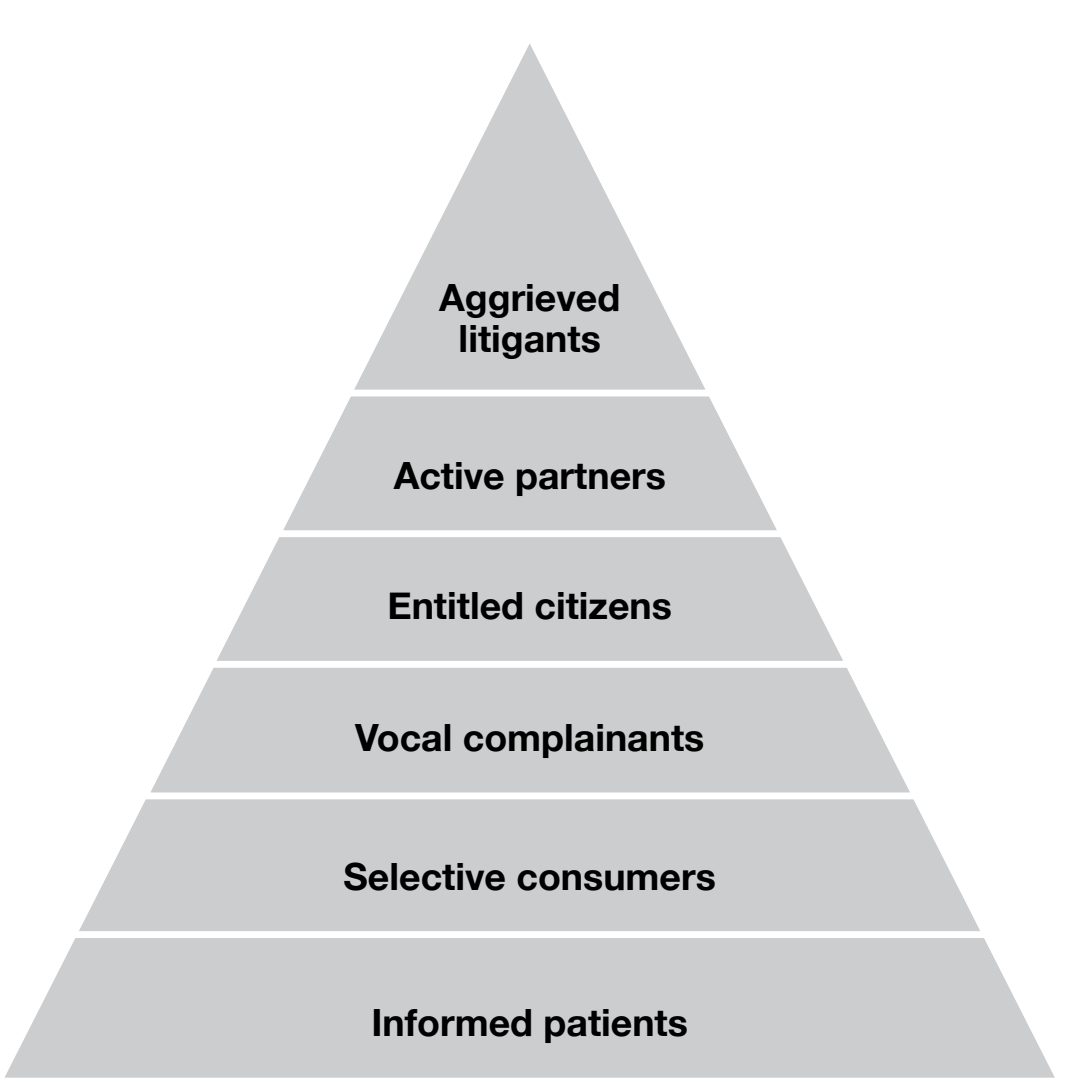

Figure 34.1 Patients as actors on a responsive regulation pyramid Source: Adapted from Healy (2011b: 285). 


\section{Informed patients}

The doctor-patient relationship is changing from the centuries-old medical tradition of 'trust me, I'm a doctor'. The doctor in this paternalistic model decided the treatment and told the patient little other than to secure compliance with the regime: 'take the antibiotics with each meal'. Patients were not asked for their views and were expected to follow instructions:

Little more than a decade ago, doctors made the decisions; patients did what they were told. Doctors did not consult patients about their desires and priorities, and routinely withheld information-sometimes crucial information, such as what drugs they were on, what treatments they were being given, and what their diagnosis was. (Gawande 2002: 2010)

Health authorities now commonly use an information strategy to empower or, at least, educate patients. This is a big task since the health services field is an example of what economists refer to as a market characterised by information asymmetry. Redressing this imbalance calls for improving the supply and accessibility of information as well as its take-up by the public. In other words, healthcare providers need to be better communicators, while patients need to be more health literate. Health literacy is a crucial attribute if people are to make informed decisions (Nutbeam 2008). Defined as the capacity to acquire, understand and use information for health, health literacy is a risk factor in poor health outcomes: such patients have higher risks of hospitalisation, have longer hospital stays, are sicker when they seek medical help, are less likely to comply with treatment and are more likely to make errors with their medication (Institute of Medicine 2004). In other words, poorly informed patients experience lower-quality and less safe health care. Recommendations on reforms to the Australian healthcare system consequently include calls for action to improve health literacy.

An information strategy aims to enable patients to make informed decisions. Good professional practice calls for a doctor to explain and obtain consent before a procedure. Moreover, 'informed consent' is a legal requirement in many countries, including in Australia under common law, whereby all competent adults have the right to consent to and refuse medical treatment. If consent is not established, there may be legal consequences for health professionals. This requirement also establishes another layer of protection against adverse events, according to a systematic review of consent procedures (Shojania et al. 2001). 
Exactly what constitutes 'informed' consent to tests and treatment remains, however, a matter for conjecture, while offering a readily understood medical explanation is a challenge when people lack health literacy.

Most people can relate to the experience of coming away from a consultation with a doctor and being unclear what was said, not necessarily because the doctor did not explain, but because anxiety got in the way of asking, listening, understanding and remembering. A manual produced for health professionals by the National Health and Medical Research Council (NHMRC 2006) suggests practical strategies to engage in better two-way communication with patients. Health authorities now put out material on many health topics. Since people can be confused by myriad internet websites that offer information (and misinformation) on health conditions and treatments, and doctors complain about their patients asking for the latest quack remedy, health authorities have set up credible websites. For example, the Australian Department of Health (in partnership with state health departments) runs HealthDirect Australia, an internet gateway that offers information on health and wellbeing and provides links to other specialist websites.

\section{Selective consumers}

Policymakers promoted consumer choice in the neoliberal reforms that swept across health sectors from the 1980s onwards. Consumer exit, more than citizen voice, was the preferred demand-side regulatory mechanism. The citizen rights rationale is that people are entitled to information that can directly affect them; the informed consumer rationale is that people need reliable information on success rates and risks to choose between providers and procedures; the market rationale is that informed consumers will choose high performers and so motivate others to improve. The concept of a selective consumer involves three elements. First, people must be able to articulate what they want from health professionals and health services. Second, they must have access to information for making informed choices. Third, they must be able to leave a service and go elsewhere. While consumer choice is an important principle, there are limitations to the concept of a selective healthcare consumer. As noted earlier, relying on patients to regulate their own health care may not be feasible or reliable in some cases: 
Hospital director: We are a major trauma hospital and patients aren't competent in much of our emergency work. In some we don't even get consent because they are straight to theatre. Also, most of our patients are really sick. Second, if a patient has something that is not pretty simple, it's difficult to get them to tell you that back reliably. Also, I don't think many patients are confident enough to challenge professionals. Unless you are part of the health culture, it's difficult to question professional advice; people just tend not to do it. (Healy 2008)

Choice is a key consumer principle that is expected to push health services to improve. Patient choice of doctor is a strongly held principle in some countries, including Australia, whereas in some others people are expected to enroll with a particular general practitioner (GP) practice. The extent of patient choice in relation to specialists and hospitals also varies. In some countries, including Australia, patients must have a referral from a GP to qualify for reimbursement for specialist care, while, in other countries, patients can bypass a GP gatekeeper and go directly to specialists.

Some governments use patient choice as a demand-side regulatory mechanism, since the economic rationalist assumption is that patients will choose high performers and so put pressure to improve on poor performers. The consumer choice principle assumes, however, that patients can make an informed choice. But it is not easy for a person to access information on which to make a choice. One method is access by the public to a medical register, which is the norm in many countries. For example, the Federation of State Medical Boards in the United States posts information on a doctor's full disciplinary history. The public has access to much less information on medical practitioners in the Australian states (Healy et al.2008). This has been improved under the Health Practitioner Regulation National Law 2009 (Cth), whereby a national register for each of the 14 health professions has been set up that offers the public access to information on practitioners including their qualifications and any restrictions or conditions placed on practice by a board or tribunal.

Public reporting is being widely adopted as a promising regulatory strategy. For example, government agencies in the United States and the United Kingdom post information on hospital scores, surgery success rates and adverse events, as do private sector agencies, such as the US Leapfrog Group and the UK 'Dr Foster' website. In Australia, state health departments have been slow to publish performance indicators 
for their public hospitals. The Australian Government in 2010 set up the MyHospitals website, with performance measures now being developed further by the National Health Performance Authority. While there is a strong public interest case, public reporting so far appears to have had a limited impact on people choosing better-performing health services. Public reporting does have an impact, however, as systematic reviews have found that reputational pressure exerts a significant impact on provider behaviour, since hospitals and surgeons care greatly about their reputations (Marshall et al. 2004).

What might be the reasons for the lack of impact on patient choice? First, it is early days for public reporting and the public is not yet familiar with this approach. Second, the information presented is usually too complex and geared more to professionals than to the public. Third, people may opt for convenience and familiarity in going to the nearest or known provider. Fourth, people are suffering from information overload since so many providers do so much public reporting-mostly glossy, confusing and self-serving-such as banks, electricity companies and schools. Finally, the public trusts hospitals and doctors, or else trusts the health authorities to regulate where necessary. Public reporting is likely to have more impact in future as the concept of performance indicators becomes better understood, and as people become more discerning users of health care given greater public knowledge about the variability of quality and safety.

\section{Vocal complainants}

Patient 'voice'-that is, the expressed views of patients-can exert pressure to improve the safety and quality of health care, with two main avenues being consumer surveys and formal complaints procedures. Patient opinion now is regarded as a legitimate indicator, among other measures, of healthcare quality. Patient satisfaction is a measure of quality from the patient's perspective and so offers a different view to collections of data from health service managers and practitioners. Patient satisfaction surveys have become the norm in many health services-although people are wearying of being asked by all manner of companies to rate an encounter. Australian state health departments now publish the results of patient surveys across different hospitals, although consumer surveys must be interpreted with caution since an overall satisfaction question generally obtains a satisfaction rating of 
more than 80 per cent. While consumer surveys arguably are a relatively reliable way of gauging patient views, anecdotal accounts can be telling. While many are testimonials by grateful patients, others tell tales of poor treatment. The personal stories of patients who experienced a medical error can be a powerful way of influencing policymakers.

All public hospitals in Australia have internal complaints procedures, as required for over a decade by intergovernmental hospital funding agreements, although hospital brochures prefer to avoid the word 'complaint' and instead ask 'what would you like to tell us?'. People can complain to a designated staff member or can take their complaint outside to an independent agency: each state and territory has a healthcare complaints commissioner or similar title (Walton et al. 2012). For example, in 2013-14, the NSW Health Care Complaints Commissioner received 4,767 written complaints, investigated 226 , took 14 to a professional standards committee and prosecuted 67 matters.

\section{Entitled citizens}

The fundamental right of citizens to good health care is set out in some countries in a constitution, bill of rights, legislation or codes of practice. Such formal entitlements offer leverage in demanding quality health care. The idea of patient rights flows from the United Nations (UN) 1948 Universal Declaration of Human Rights in that a person has a fundamental right to good health and to good health care. Views on patients' rights differ across countries depending on social and political norms, and there are still some echoes of attitudes depicted in Solzhenitsyn's novel Cancer Ward, in which patients had to battle with the doctors for information about their treatment:

[Doctor] It's strictly against the rules for patients to read medical books ...

[Patient] What is the diagnosis?

[Doctor] Generally speaking, we don't have to tell our patients what's wrong with them ... (Solzhenitsyn 2003: 41)

Since at least the 1980s in Australia, the state has been expected to consult with its citizens on matters that affect them. Patient participation has been adopted in the health sector and health managers are expected to be adept in managing consultation with patients and many other 
vested interests, including patient advocacy groups (Dugdale 2008: 194). Major changes in health policy are generally not undertaken without a round of consultations.

What sort of standards can patients reasonably expect from their health services? Both patients and providers need some guidance. 'Patient charters' are produced that encapsulate such standards rather than legally enforceable 'rights'. The Australian Charter of Healthcare Rights, endorsed by the country's health ministers in 2008, required the states to ensure that their public hospitals post a patient charter. A charter must inform patients they are entitled to free access to public hospital treatment, to be treated as a public or a private patient within a public hospital, set out the process by which patients can complain about hospital services and explain how complaints can be made to an independent complaints body.

Nordic countries led the way in passing patients' rights legislationfor example, Norway passed the Act on Patients' Rights 1999, which included the right to choose a hospital, to have access to a specialist evaluation within 30 days of referral, to a second opinion, to be fully informed, to give informed consent and to have access to complaints procedures. New Zealand also stresses a rights approach in its Code of Health and Disability Services Consumers' Rights. Australia does not have a 'bill of rights' (except in the Australian Capital Territory) in relation to citizen rights in general or to patients' rights in particular, and no legislation at national or state level sets out a comprehensive statement of patient rights. The law in Australia has concerned itself with specific aspects, such as ensuring that a person gives consent to health care, breach of contract between a doctor and a patient involving medical negligence and a person's right to access their own medical information (McIlwraith and Madden 2006).

Although many countries have informed consent legislation, patients are not necessarily legally entitled to know when an error occurs in their medical treatment. Many studies have found that most patients are not told about a medical error and that most believe they should be told (Studdert 2009). The Australian Commission on Safety and Quality in Health Care advocates that health professionals tell their patients when something goes wrong and has produced guidance on 'open disclosure'. 


\section{Active partners}

Citizen participation strategies are pursued in the health sector with the intention of redressing the unequal power relationship between professionals and patients. The citizen participation concept suggests a democratic paradigm where a person is an active partner in decisionmaking. This approach aligns with community development principles that broadly are about removing conditions of domination (for example, by health professionals) and increasing the self-determination of individuals by lifting restrictions (for example, by providing accessible and good health care) and by increasing capacity (for example, through information and empowerment). In transactions between people and health services, the therapeutic argument is that involving patients as partners in the process results in satisfied patients and in better health outcomes. The quality argument is that patients understand their own needs best and can help ensure the safety and quality of their own care. The governance argument is that consumers can regulate their own health care: in market terms, they are coproducers of their own health care and, in citizenship terms, they are active participants.

Most health sector boards and advisory councils now include consumer representatives, but the issue of who best represents healthcare users remains problematic. Boards are more likely to seek members from consumer organisations (substantive representation) than to aim for a microcosm of the consumer population (descriptive representation) or to seek members through a democratic voting procedure (formal representation).

The claim is that research shows that patients who are more involved with their care tend to get better results (Agency for Healthcare Research and Quality 2008). The evidence for this claim is strong, particularly in the area of chronic care management, where people have a long-term commitment to understanding and being involved in the management of their own health care. The World Health Organization (WHO) World Alliance for Patient Safety has set up an international network of patient organisations to enlist consumers in helping to drive the patient safety movement. Health services now enlist patients as partners in a range of activities that involve them in contributing to treatment decisions, checking the accuracy of records and processes, monitoring their own treatment and being involved in self-management (Coulter and Ellins 2007). For example, people now have the right to read their own medical record, which previously was regarded as the property of the healthcare 
provider; and asking patients to verify information is an effective safety check given the many errors that can creep into medical records. Patients increasingly are involved in monitoring and managing their own health, particularly those with chronic conditions such as asthma and diabetes.

While people, in theory, can be active partners, in practice, many, or perhaps most, need to be reassured that it is okay to question, let alone challenge, doctors and nurses. This reticence by patients is deeply embedded in the healthcare culture. Patients are reluctant, for example, to remind doctors and nurses that they should wash their hands before physically examining them. For example, a survey of patients in English public hospitals found that although 71 per cent said that patients should be involved in improving hand hygiene, only 26 per cent said they were willing to remind staff to clean their hands (Davis et al. 2008). Some countries produce brochures to encourage people to speak up. For example, a US brochure, 'Ask Me 3', urges patients to ask at least three questions during a visit to the doctor: 'What is my main problem? What do I need to do? Why is it important for me to do this?' The Australian Commission on Safety and Quality in Health Care puts out 'Ten Tips for Safer Health Care', which exhorts people to 'speak up if you have any questions or concerns'.

Stephen Schneider, who was diagnosed with a rare type of lymphoma, is an example of a patient who was an active partner in discussing and deciding his treatment decisions with top US clinicians (Schneider 2005). The title of his book says it all: The Patient from Hell: How I Worked with My Doctors to Get the Best of Modern Medicine and How You Can Too. He acknowledged, however, that he was better qualified than the average patient to research the condition and treatment options, and that many patients may need a knowledgeable advocate to help them on their patient journey.

There are limits to the regulatory power of an individual, of course, so the concept of networked regulation suggests ways that one person can enlist other people and groups in a regulatory enterprise (Braithwaite 2009). One of the reasons that people entrust others-whether governments or health insurance funds- to purchase health services on their behalf is that it is difficult for a lay person to make an informed decision on a procedure, doctor or hospital, because this often requires considerable medical and technical knowledge, so it is easier to trust one's doctor to make the decision (Dugdale 2008: 132). The role of health broker in negotiating decisions is undertaken by a person's general physician in many health systems, and by a health maintenance organisation in the 
case of the US private health insurance model. The increasing complexity of health care has prompted the role of a trusted intermediary (broker, case manager, advocate) who can manage interactions with health services and insurance funds on behalf of a patient.

The strategy of enlisting others with greater powers also aligns with the idea of nodal governance, whereby a regulator seeks to exert influence through a concentration of regulators at a particular location in a regulatory field (Burris et al. 2005; Holley and Shearing, Chapter 10, this volume). For example, the Australian Commission on Safety and Quality in Health Care gathers together experts and policymakers as leaders on strategies to improve health care. A patient can recruit more powerful actors who have the capacity to connect together and pull the various strands of power. An individual patient may therefore exert influence through a better informed family member or friend, a health ombudsman or lawyer, or through groups that range from self-help groups based on mutual assistance between peers to formal organisations run by a board and employing professional staff. Large non-governmental organisations (NGOs) advocate on behalf of their clients or on behalf of a defined population group and might also engage in research, prevention and treatment.

\section{Aggrieved litigants}

Some observers propose 'regulation by litigation' as an effective strategy for regulating health care. Compensation for medical injuries in common law countries, including Australia, is a fault system based mainly on the tort of negligence-torts being civil wrongs where compensation is sought. Several categories of charges by patients against health professionals can arise, including negligence and criminal charges, such as assault (McIlwraith and Madden 2006). The main arguments for patients being able to sue healthcare providers are that it is a necessary avenue of last resort for aggrieved patients and, second, that it has a salutary impact in ensuring better and safer health care (Hirsch 2009). The counter view, however, is that fear of being sued makes health professionals more likely to cover up rather than learn from their mistakes, and that the experience of litigation is traumatic and costly for all concerned. Further, since many cases are settled out of court, the opportunity for wider learning is limited, since medical indemnity insurance funds are not keen to publicise and so promote more litigation by patients. 


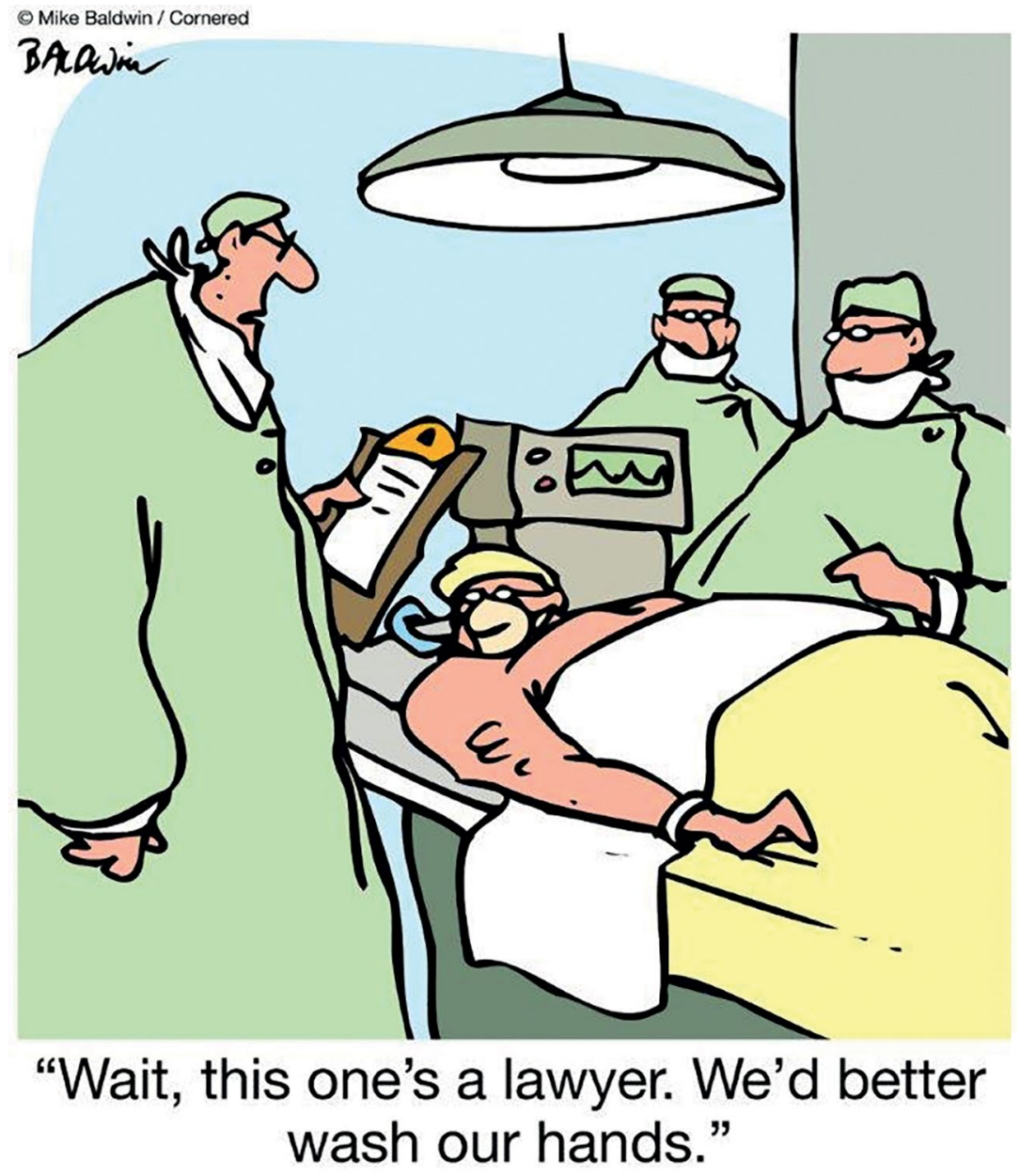

Figure 34.2 The salutary threat of litigation Source: CartoonStock.

\section{Concepts and conclusions}

The responsive regulation model works well as a predictor and working model when applied to the distinctive and complex context, culture and behaviour of health professionals. While much of the regulation literature focuses on regulatory authorities, the responsive regulation model developed by John Braithwaite and colleagues broadens this perspective to encompass a variety of actors (state and non-state) and a variety of mechanisms (from persuasion to enforcement). Regulation from this 
perspective is defined broadly as steering the flow of events (Ayres and Braithwaite 1992). The pyramidal framework promotes the view that it is better to begin with respectful and cheaper strategies at the base. This allows virtuous actors to voluntarily comply with reminders about standards, while rational actors calculate that it is in their interests to comply. This makes sense in relation to the health sector since the great majority of health professionals seek to do good, not harm. The main point here, however, in relation to patients as regulators is that patients must be able to escalate from softer to stronger actions (both supports and sanctions) if required.

The two pyramids—supports-based and sanctions-based-offer a more nuanced framework for how patients can support better health care and how they can invoke sanctions to insist on compliance with standards and to punish malpractice. The concepts of networked and nodal governance are important in that they offer empowerment strategies whereby individual patients recruit others to strengthen their influence over the health system.

The behaviour of patients as regulators can also be depicted in terms of a ladder of actions. A classic paper by Arnstein (1969) applied a power analysis to citizen participation in depicting a ladder with eight rungs, beginning with the bottom rung of manipulation and ascending upwards through therapy, information provision, consultation, placation, partnership and delegated power to the top rung of citizen control. While most people are willing to step on to the bottom rung to answer a consumer survey, very few want to climb to the top rungs or become a board member. This changes the idea of a ladder to something more resembling a pyramidal shape.

There has been a dramatic change in the nature of the encounter between health professionals and patients; the days are gone when patients hardly dared question their doctor. Admittedly, we have to be quick with our questions given the usual 10-minute GP consultation. The policy thrust, at least in well-developed healthcare systems, is to promote the ways patients/the public can have more say and to require health services to be more transparent in reporting on their performance. While patients are wise to be more cautious, the counter argument is that patients must be able to trust their doctors in a crisis, and, further, trust in one's doctor is said to have a therapeutic effect (Mechanic and Meyer 2000). 
What is the evidence that patients can be effective regulators? Research on patients as regulatory actors remains sketchy, with few rigorous systematic reviews so far undertaken. While there is considerable research on methods to impart information and to improve patient health literacy, a structured review concluded that the role of patients as regulatory actors was still in its early stages and that safety outcomes were difficult to measure (Coulter and Ellins 2007). The available evidence for the different strategies can be summarised as follows:

- Informed patients (that is, people who are health literate) have better health outcomes.

- Selective consumers have had little impact but this may strengthen with better public reporting on health services' performance.

- Vocal complainants can secure individual redress but it is unclear to what extent systemic improvements follow.

- Entitled citizens are well accepted in democratic societies and legislation and codes of patient rights are arguably influential in improving service delivery.

- Active partners who are involved in decisions on their own health care have better outcomes, as shown in studies of chronic disease management.

- Aggrieved litigants can secure compensation but some argue that defensive medicine is a barrier to quality medicine.

\section{Further reading}

Australian Commission on Safety and Quality in Health Care 2011. Patient-Centred Care: Improving Quality and Safety through Partnerships with Patients and Consumers. Sydney: ACSQHC. Available at: safetyandquality.gov.au/wp-content/uploads/2012/01/ PCCC-DiscussPaper.pdf.

Iedema, R, Allen, S, Britton, K, Piper, D, Baker, A and Grbich, C 2011. 'Patients' and family members' views on how clinicians enact and how they should enact incident disclosure: The "100 patient stories" qualitative study', British Medical Journal 343: 1-9. doi.org/10.1136/ bmj.d4423. 
Stewart, E 2013. 'What is the point of citizen participation in health care?', Journal of Health Services Research E Policy 18(2): 124-6. doi. org/10.1177/1355819613485670.

\section{References}

Agency for Healthcare Research and Quality 2008. National Healthcare Quality Report 2007. Rockville, Md: Agency for Healthcare Research and Quality.

Arnstein, S 1969. 'A ladder of citizen participation', American Institute of Planners Journal 35(4): 216-24. doi. org/10.1080/01944366908977225.

Ayres, I and Braithwaite, J 1992. Responsive Regulation: Transcending the Regulation Debate. Oxford: Oxford University Press.

Braithwaite, J 2009. 'Leading from behind with plural regulation', in J Healy and P Dugdale (eds), Patient Safety First: Responsive Regulation in Health Care. Sydney: Allen \& Unwin, pp. 24-43.

Braithwaite, J, Makkai, T and Braithwaite, V 2007. Regulating Aged Care: Ritualism and the New Pyramid. Cheltenham, UK: Edward Elgar. doi.org/10.4337/9781847206855.

Burris, S, Drahos, P and Shearing, C 2005. 'Nodal governance', Australian Journal of Legal Philosophy 30: 30-58.

Coulter, A and Ellins, J 2007. 'Effectiveness of strategies for informing, educating and involving patients', British Medical Journal 335: 24-7. doi.org/10.1136/bmj.39246.581169.80.

Davis, R, Koutantji, M and Vincent, CA 2008. 'How willing are patients to question healthcare staff on issues related to the quality and safety of their healthcare? An exploratory study', Quality and Safety in Health Care 17(2): 90-6. doi.org/10.1136/qshc.2007.023754.

Dugdale, P 2008. Doing Health Policy in Australia. Sydney: Allen \& Unwin.

Gawande, A 2002. Complications: A Surgeon's Notes on an Imperfect Science. New York: Metropolitan Books, Henry Holt \& Co. 
Healy, J 2008. Safe surgery in Australian hospitals: Implementation of the correct patient, correct site, correct procedure protocol, RegNet Occasional Paper No. 12, Regulatory Institutions Network, Canberra. Available at: regnet.anu.edu.au/sites/default/files/ publications/attachments/2015-05/ROP12b_0_0.pdf.

Healy, J 2011a. 'Implementing a safe surgery protocol in Australian hospitals through responsive regulation', International Journal on Quality in Health Care 24(1): 1-7.

Healy, J 2011b. Improving Health Care Safety and Quality: Reluctant Regulators. Farnham, UK: Ashgate.

Healy, J and Dugdale, P (eds) 2009. Patient Safety First: Responsive Regulation in Health Care. Sydney: Allen \& Unwin.

Healy, J, Maffi, C and Dugdale, P 2008. 'A national medical register: Balancing public transparency and professional privacy', Medical Journal of Australia 188(4): 247-9.

Hirsch, D 2009. 'Does litigation against doctors and hospitals improve quality?', in J Healy and P Dugdale (eds), Patient Safety First: Responsive Regulation in Health Care. Sydney: Allen \& Unwin, pp. 254-72.

Hirschman, A 1970. Exit, Voice and Loyalty: Responses to Decline in Firms, Organizations and States. Cambridge, Mass.: Harvard University Press.

Institute of Medicine 2004. Patient Safety: Achieving a New Standard of Care. Washington, DC: The National Academies Press.

McGlynn, E, Asch, SM, Adams, J, Keesey, J, Hicks, J, DeCristofaro, A and Keer, EA 2003. 'The quality of health care delivered to adults in the United States', New England Journal of Medicine 348(26): 2635-45. doi.org/10.1056/NEJMsa022615.

McIlwraith, J and Madden, B 2006. Health Care and the Law. Sydney: Law Book Company.

Marshall, M, Romano, PS and Davies, HTO 2004. 'How do we maximize the impact of the public reporting of quality of care?', International Journal for Quality in Health Care 16(Supp. 1): i57-i63. doi.org/10.1093/intqhe/mzh013. 
Mechanic, D and Meyer, S 2000. 'Concepts of trust among patients with serious illness', Social Science and Medicine 51(5): 657-68. doi. org/10.1016/S0277-9536(00)00014-9.

Nutbeam,D 2008.'The evolving concept of health literacy', Social Science \& Medicine 67(12): 2072-8. doi.org/10.1016/j.socscimed.2008.09.050.

Runciman, W, Webb, RK, Helps, SC, Thomas, EJ, Sexton, EJ, Studdert, DM and Brennan, TA 2000. 'A comparison of iatrogenic injury studies in Australia and the USA', International Journal of Quality in Health Care 12(5): 379-88. doi.org/10.1093/intqhe/12.5.379.

Schneider, S 2005. The Patient from Hell: How I Worked with My Doctors to Get the Best of Modern Medicine and How You Can Too. Cambridge, Mass.: Perseus Books.

Shojania, KG, Duncan, BW, McDonald, KM and Wachter, RM 2001. Making Health Care Safer: A Critical Analysis of Patient Safety Practices. Rockville, Md: Agency for Healthcare Research and Quality. Available at: archive.ahrq.gov/clinic/tp/ptsaftp.htm.

Solzhenitsyn, A 2003. Cancer Ward. London: Vintage Classics, Random House.

Studdert, D 2009. 'Disclosure of medical injury', in J Healy and P Dugdale (eds), Patient Safety First: Responsive Regulation in Health Care. Sydney: Allen \& Unwin, pp. 237-53.

Walton, M, Smith-Merry, J, Healy, J and McDonald, F 2012. 'Health complaint commissions in Australia: Time for a national approach to data collection', Australian Review of Public Affairs 11(1): 1-18. 
This text is taken from Regulatory Theory: Foundations and applications, edited by Peter Drahos, published 2017 by ANU Press, The Australian National University, Canberra, Australia. 